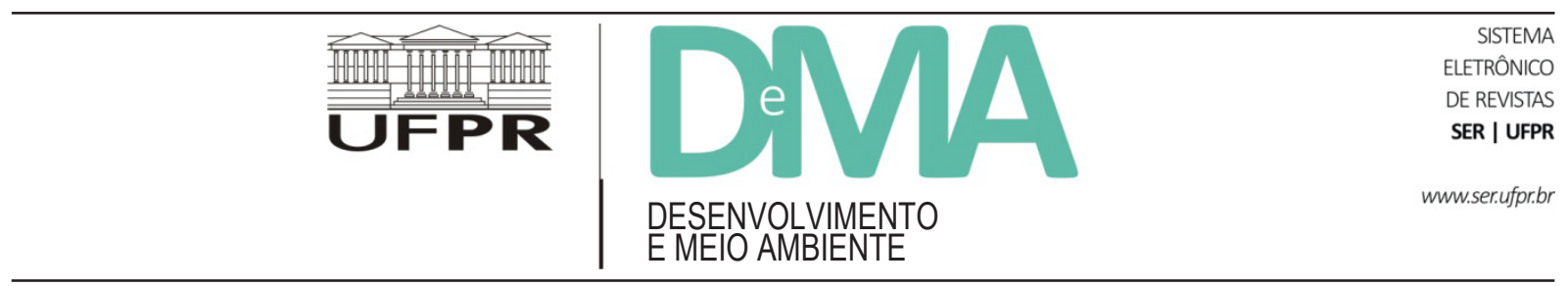

\title{
Inclusão dos serviços ecossistêmicos em estudos de impacto ambiental: evidência empírica no estado de São Paulo
}

\section{Ecosystem Services Input in Environmental Impact Statements: Empirical Evidence in the State of São Paulo, Brazil}

\author{
Moema Pauline Barão SEPTANIL ${ }^{1 *}$, Lucila PINTO ${ }^{1}$, Ligia Maria Barrios CAMPANHÃO1 \\ ${ }^{1}$ Escola de Engenharia de São Carlos, Universidade de São Paulo (USP), São Carlos, SP, Brasil. \\ *E-mail de contato: moema@usp.br
}

Artigo recebido em 27 de julho de 2017, versão final aceita em 14 de novembro de 2017.

RESUMO: Diante do cenário recente de uso insustentável e degradação dos serviços ecossistêmicos (SEs), a Avaliação de Impacto Ambiental (AIA), em razão de seu caráter preventivo, tem o potencial de introduzir SEs na tomada de decisão. Todavia, poucos estudos exploraram essa vinculação. Assim, o presente trabalho buscou avaliar a inclusão do conceito de serviços ecossistêmicos no processo de AIA, por meio da análise de Estudos de Impacto Ambiental (EIAs) no estado de São Paulo. Para isso, foram selecionados 110 EIAs entre 2006 e 2014, nos quais foram feitas buscas por palavras-chave relativas aos SEs. Os EIAs enquadrados foram submetidos a uma análise sobre a incorporação dos SEs nos EIAs com base em 12 critérios, cuja pontuação possibilitou o cálculo de Índices de Análise dos Serviços Ecossistêmicos (IASE). Dos 110 estudos analisados, 20 apresentaram termos relativos ao conceito de SEs. A adoção do conceito foi instável entre 2006 e 2010, mas, a partir de 2011, houve um aumento no uso dos termos, que parece ter sido voluntária. Apesar desse resultado, os EIAs que citaram os SEs não os incorporaram adequadamente à análise, ou seja, os SEs foram fracamente identificados, e os EIAs em geral não apontaram possíveis impactos nestes serviços e não previram medidas mitigadoras exclusivamente resultantes dessa análise direcionada. Apesar de mais frequente, o conceito de SEs ainda não está incorporado à prática de AIA no estado de São Paulo, o que sugere a necessidade de políticas mais dedicadas a esse fim.

Palavras-chave: estudo de impacto ambiental; serviços ecossistêmicos; serviços ambientais; São Paulo; Brasil.

ABSTRACT: Facing the recent scenario of unsustainable use and degradation of ecosystem services (ESs), Environmental Impact Assessment (EIA) is a preventive instrument to introduce ESs in decision-making. This research aimed to assess the ESs input in the EIA process through the analysis of Environmental Impact Statements (EISs) in São Paulo state, Brazil. For that reason, one hundred and ten EISs from 2006 to 2014 were selected and searches for keywords related to ESs were done. A systematic review process examined the EISs containing the keywords based on a 12 criteria review and using an 'Ecosystem Service Assessment Index' (IASE). Twenty EISs presented keywords related to ESs, and while the adoption of this concept was unstable between 2006 and 
2010, from 2011 the concept input increased, apparently in a voluntary way. Despite this result, those EISs did not adequately address ESs into the EIA process: ESs were poorly identified and few EISs detected potential impacts on the ESs and provided mitigation measures. Despite being more frequent in the EISs, the concept of ESs is not included in the EIA practice in São Paulo state, which suggests the need for explicit policies for this purpose.

Keywords: environmental impact statement; ecosystem services; environmental services; São Paulo; Brazil.

\section{Introdução}

Os serviços ecossistêmicos (SEs) são os benefícios que a humanidade obtém dos ecossistemas. Tais serviços incluem, além dos tradicionais produtos econômicos, outras funções que atuam no suporte à vida humana, como a ciclagem de nutrientes, a regulação climática, a mitigação de enchentes, dentre outras (Daily, 1997). Segundo o MEA (2005a), os SEs são classificados em diferentes categorias, a saber: serviços de suporte, de provisão, de regulação, e culturais, sendo que todos eles se relacionam direta e indiretamente com os componentes do bem-estar humano.

Entretanto, nos últimos 50 anos, as atividades humanas vêm se intensificando, causando maiores danos aos serviços supracitados. Em 2005, cerca de $60 \%$ dos SEs se encontravam degradados ou sendo utilizados de forma insustentável, e é previsto que os efeitos negativos oriundos da degradação destes serviços se intensifiquem nos próximos 50 anos (MEA, 2005a). Alguns impactos decorrentes desta degradação dos SEs podem afetar direta e indiretamente a saúde humana, tais como as inundações, a escassez de água e a exposição a poluentes (MEA, 2005b).

Frente a isso, conservar os SEs e barrar sua degradação ainda é um grande desafio, mas que pode ser alcançado por meio da consideração dos SEs pelas políticas, instituições e práticas (Daily et al., 2009). Esta afirmação vai ao encontro da abordagem ecossistêmica, vista como estratégica para a conservação da biodiversidade e dos recursos naturais, preconizada pela Convenção sobre a Diversidade Biológica (CDB, 2015).

A Avaliação de Impacto, de acordo com a International Association for Impact Assessment (Associação Internacional de Avaliação de Impacto - IAIA), pode ser definida no seu conceito mais simples como o processo de identificar as consequências futuras de ações propostas. Ainda, a Avaliação de Impacto Ambiental (AIA) é o processo de identificar, predizer, avaliar e mitigar os efeitos biofísicos, sociais e outros efeitos relevantes antes da tomada de decisão e de se assumirem compromissos (IAIA, 2009). Frente a isso, um dos objetivos principais da AIA é dar suporte à tomada de decisão, já que este processo oferece previamente uma análise sistemática das implicâncias ambientais de uma ação e suas alternativas (Glasson et al., 2012).

No Brasil, um dos documentos associados à AIA e ao licenciamento ambiental é o Estudo de Impacto Ambiental (EIA), que é considerado a atividade central do processo de AIA (Sánchez, 2013), sobretudo de grandes projetos. O EIA é definido como um estudo técnico de caráter interdisciplinar que tem como finalidade predizer, identificar, valorizar e corrigir as consequências ou efeitos ambientais que as ações dos empreendimentos possam causar sobre a qualidade de vida do homem e do meio (Conesa Fernández-Vítora, 2006). A norma brasileira que estabelece o conteúdo de um EIA afirma que o 
mesmo deve englobar: um diagnóstico ambiental da área de influência, a análise dos impactos ambientais do projeto e de suas alternativas, a definição das medidas mitigadoras dos impactos negativos e a elaboração do programa de acompanhamento e monitoramento dos impactos positivos e negativos (CONAMA, 1986).

Diante do cenário de degradação dos SEs e do caráter preventivo e de apoio à tomada de decisão característico da AIA, este instrumento tem o potencial de introduzir questões relacionadas aos SEs na tomada de decisão, o que ainda se mostra limitado (Geneletti, 2013, Laurans et al., 2013). Uma vantagem associada ao emprego do conceito de SEs é a possibilidade de conectar de forma mais explícita a importância de determinados ecossistemas com a produção de bens e benefícios que são valorados positivamente pelas pessoas (Karjalainen et al., 2013), fortalecendo os ganhos e minimizando as perdas para os beneficiários (Honrado et al., 2013). Assim, é possível ilustrar explicitamente as consequências socioeconômicas de um determinado desenvolvimento em uma paisagem, propiciando que os SEs contribuam positivamente para a tomada de decisão (Tardieu et al., 2015).

A inclusão dos SEs tem potencial para superar alguns dos problemas correntes na prática da AIA (Rosa \& Sánchez, 2016) ao contribuir para elaborar escopos mais eficientes; permitir o engajamento de partes interessadas, especialmente comunidades afetadas; proporcionar a elaboração de uma baseline mais integrada; contribuir para identificação mais completa dos potenciais impactos; propiciar a avaliação dos impactos na perspectiva de comunidades afetadas; e contribuir para a concepção de propostas de mitigação mais proporcionais à importância dos impactos (Rosa \& Sánchez, 2015, 2016). Entretanto, a incorporação dos SEs deve ser feita de forma específica ao contexto em que está sendo aplicado o instrumento (Baker et al., 2013; Rosa \& Sánchez, 2015). Além disso, a potencial contribuição dos SEs à prática da AIA dependerá de como os envolvidos percebem as limitações e potencialidades desta abordagem (Baker et al., 2013; Rosa \& Sánchez, 2015). É necessário conhecer as preferências das partes interessadas, assim como os eventuais trade-offs entre os benefícios providos pelos SEs e os custos e perdas referentes às alternativas (Karjalainen et al., 2013).

De acordo com Landsberg et al. (2011), os SEs podem ser inseridos no processo da AIA em três etapas: escopo, análise dos impactos e mitigação dos impactos, sendo que estas duas últimas se encontram inseridas no processo de elaboração dos EIAs. A inclusão dos SEs no escopo permite identificar aqueles serviços que podem ser impactados, enquanto que a segunda etapa permite que os SEs identificados e priorizados sejam avaliados quanto aos possíveis impactos. Já na etapa de mitigação, a consideração dos SEs pela mesma pode garantir que o desenvolvimento do projeto e as medidas de gestão considerem os principais beneficiários locais dos SEs (Rosa \& Sánchez, 2016; Mandle et al., 2016).

Diante do exposto, a inserção da análise dos SEs nos EIAs desponta como um elemento para potencializar a predição e a respectiva mitigação da degradação ambiental. Diante disto, este trabalho buscou avaliar a inclusão do conceito de serviços ecossistêmicos no processo de Avaliação de Impacto Ambiental, especificamente no conteúdo de Estudos de Impacto Ambiental submetidos a processos de licenciamento ambiental no estado de São Paulo. Para isso, conforme exposto nas próximas seções, primeiramente foi verificada a inclusão dos termos relativos a serviços ecossistêmicos nos EIAs submetidos ao órgão ambiental responsável pelo licenciamento no estado de São Paulo entre os anos de 2006 e 2014. Posteriormente, analisou-se como o tema 
serviços ecossistêmicos tem sido incorporado na análise ambiental dos EIAs que incluíram o conceito.

\section{Metodologia}

\subsection{Seleção da amostra e busca pelas palavras-chave}

Para o desenvolvimento deste trabalho, foi adotado como universo de estudo os EIAs submetidos à Companhia Ambiental do Estado de São Paulo (CETESB), órgão responsável pelo licenciamento ambiental no estado. Inicialmente foram selecionados 122 EIAs entre 2006 e 2014, obtidos diretamente do sítio eletrônico da CETESB (CETESB, 2015). A seleção de documentos a partir do ano de 2006 se deu em razão da publicação do relatório Millennium Ecosystem Assessment em 2005, que avaliou a situação dos SEs e apresentou o quadro de degradação dos serviços descrito anteriormente. A consciência acerca da necessidade de conservar os SEs e a pesquisa neste tema já eram crescentes, entretanto, tal relatório pode ser considerado responsável por introduzir mais incisivamente os SEs no pensamento técnico, científico e político (Honrado et al., 2013). Dessa forma, foi considerada como mais provável a ocorrência dos termos buscados em estudos dos processos elaborados a partir de 2006 até 2014, último ano cujos processos estiveram disponíveis para consulta durante o desenvolvimento desta pesquisa.

De posse da amostra de EIAs, foi feita uma busca por termos relacionados aos SEs, a saber: "serviço ambiental", "serviços ambientais", "serviço ecossistêmico", "serviços ecossistêmicos". A seleção das palavras-chave "serviços ecossistêmicos" e "serviços ambientais" foi feita em razão da discussão sobre a diferença terminológica destes conceitos (Wunder, 2005). Para o propósito deste trabalho, ambos foram considerados como sinônimos, principalmente porque o termo "serviços ambientais" é amplamente utilizado na literatura nacional em português e poderia ser mais frequentemente empregado pelos relatórios analisados.

\subsection{Análise da incorporação dos serviços ecossistêmicos nos EIAs}

A segunda etapa correspondeu à análise da incorporação dos SEs nos Estudos de Impacto Ambiental que apresentaram as palavras-chave, verificando se determinadas tarefas foram cumpridas. Essa etapa buscou investigar a inclusão explícita dos SEs na análise ambiental, ou seja, utilizando termos como serviços ambientais, serviços ecossistêmicos ou mesmo serviços providos pelos ecossistemas. Para isso, foram selecionados 12 critérios de análise, extraídos do trabalho de Rosa \& Sánchez (2015), que estão listados na Tabela 1.

TABELA 1 - Critérios de análise do conteúdo dos EIAs

\begin{tabular}{l} 
Questões para a análise da incorporação dos SEs no \\
EIA \\
\hline 1. Os ecossistemas afetados estão descritos em um capítulo \\
dedicado aos SEs? \\
2. Há identificação dos SEs potencialmente afetados? \\
3. Os beneficiários de cada SE estão descritos? \\
4. Os SEs estão priorizados? \\
5. Há uma delimitação da área de estudo para os SEs? \\
6. Há uma baseline dedicada aos SEs prioritários? \\
7. Há alguma forma de previsão dos impactos nos SEs? \\
8. Os impactos nos SEs prioritários estão analisados? \\
9. Existem medidas de mitigação indicadas para os SEs? \\
10. Há menção dos SEs em capítulos separados daquele \\
dedicado aos SEs? \\
11. Os diferentes tipos de SEs são explicitados em Serviços \\
$\quad$ de Provisão, Serviços regulatórios, Serviços Culturais e \\
Serviços de Suporte? \\
12. Existem recomendações de mitigação resultando \\
apenas da análise dos SEs? \\
\hline FONTE. adaptado de Rosa \& Sánchez (2015)
\end{tabular}


Para cada um dos critérios, foram atribuídas as seguintes respostas e seus respectivos valores: "Adequado" (1), ou seja, o EIA aborda integralmente a questão; "Parcialmente adequado" $(0,5)$, quando há uma abordagem, mas a mesma não é completa; e "Não citado ou não realizado" (0). A partir destas respostas, foi elaborado um Índice de Análise dos Serviços Ecossistêmicos (IASE), adaptado a partir da metodologia apresentada por Atkinson et al. (2000), que buscou analisar a inclusão da biodiversidade em EIAs dos Estados Unidos. O índice foi elaborado a fim de sistematizar e facilitar a comparação da inclusão dos SEs entre os EIAs do período selecionado e também ao longo do tempo. Os valores do IASE podem variar entre 0 e 1 , sendo que quanto mais próximo de 1 , melhor a inclusão dos SEs no EIA. O cálculo do IASE foi feito da seguinte forma:

$$
\mathrm{IASE}=\frac{(1 \times \mathrm{A})+(0,5 \times \mathrm{B})}{\mathrm{n}}
$$

Onde:

IASE = Índice de Análise dos SEs do EIA

$\mathrm{A}=$ número de critérios respondidos com "Adequado"

$\mathrm{B}=$ número de critérios respondidos com "Parcialmente"

$\mathrm{n}=$ número de critérios de análise

\section{Resultados e discussão}

Dos 122 Estudos de Impacto Ambiental selecionados entre os anos 2006 e 2014, 110 documentos foram analisados, pois alguns arquivos estavam ausentes ou corrompidos, impossibilitando a análise da amostra completa. Dos 110 EIAs analisados, 25 apresentaram pelo menos uma das palavras-chave "serviço ambiental", "serviços ambientais", "ser- viço ecossistêmico" e "serviços ecossistêmicos". Entretanto, apenas 20 foram selecionados para uma análise mais detalhada, pois uma primeira aproximação indicou que as palavras-chave encontradas em cinco dos 25 EIAs não se referiam ao conceito de serviços ecossistêmicos, mas sim a nomes de empresas consultoras e prestadoras de serviços.

Os 20 EIAs analisados corresponderam a tipologias variadas de empreendimentos: transporte - rodovia e VLT (6), loteamento urbano (3), aterro sanitário e estação de tratamento/transferência de resíduos (4), barragem e pequena central hidrelétrica (3), usina de açúcar e álcool (1), mineração (1) e outros (2).

Conforme a Figura 1, nota-se que houve um aumento na inclusão destes termos nos EIAs a partir de 2011, sendo que anteriormente a essa data a incorporação não demonstrou seguir um padrão. Em 2014, a grande maioria (80\%) dos EIAs paulistas elaborados citou pelo menos uma das palavras-chave. Ao analisar 12 processos de AIA portugueses anteriores e posteriores à publicação do relatório do MEA (2005a), Honrado et al. (2013) verificaram que nenhum dos casos referenciou explicitamente os SEs em seus documentos, como conceito ou mesmo em relação às categorias do MEA (2005a) inseridas neste trabalho na questão do critério 11 da Tabela 1. De acordo com Jacob et al. (2016), os SEs não costumam ser citados explicitamente pelas normas regulatórias, entretanto, sua consideração já se encontra incluída de forma implícita na AIA, fato também verificado por Honrado et al. (2013). No caso da amostra analisada na presente pesquisa, essa incorporação dos SEs nos EIAs se mostra mais explícita do que o quadro encontrado por Honrado et al. (2013) e Jacob et al. (2016) em seus trabalhos. Cabe destacar que, como a metodologia utilizada se ateve à menção explícita dos conceitos, é possível que implicitamente um número maior de EIAs 


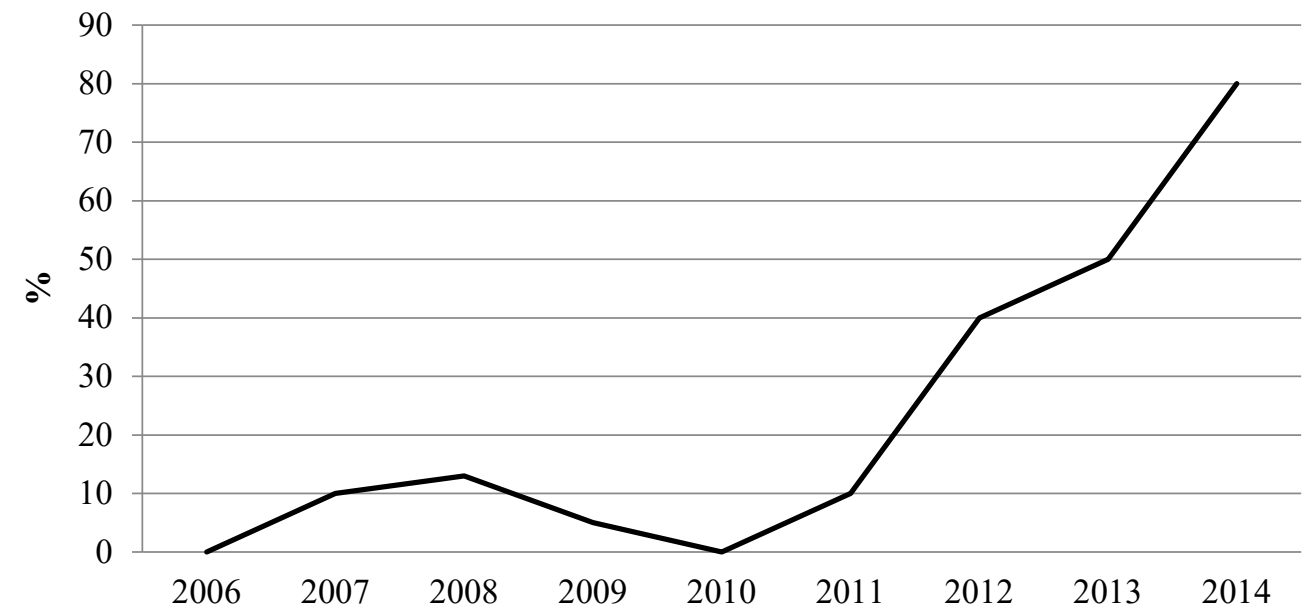

Anos

FIGURA 1 - Evolução da proporção dos EIAs que incluíram pelo menos uma das quatro palavras-chave referentes a serviços ecossistêmicos entre 2006 e 2014.

paulistas tenha citado ou avaliado esses serviços em alguma etapa do processo, embora sem referenciar o conceito que foi difundido pelo MEA (2005a).

Os resultados para a amostra paulista indicam que os responsáveis pela elaboração dos estudos ambientais passaram a incorporar os SEs de forma voluntária, visto que a diretriz mais recente derivada do órgão ambiental ainda não exigia a incorporação deste conceito na análise ambiental a ser apresentada pelo documento (CETESB, 2014). O caráter voluntário da introdução dos SEs pode também ser sugerido, pois os EIAs analisados não fazem menção aos requerimentos da International Finance Corporation (Corporação Financeira Internacional - IFC), que condiciona seus investimentos à aplicação dos seus Padrões de Desempenhos (Performance Standards on Environmental and Social Sustainability - PS), cujo PS $n^{0} 6$ exige a avaliação dos SEs. Ainda, orientações metodológicas para a incorporação dos SEs providas pela IFC (IFC, 2012) e pelas guias do World Resources
Institute (Instituto de Recursos Mundiais - WRI) (Landsberg et al., 2013) não foram referenciadas pelos EIAs. Essa tendência voluntária de integração dos SEs pelos EIAs não foi encontrada por Rosa \& Sánchez (2015) visto que, ao investigar relatórios de avaliação de impacto na África, Ásia e América do Sul, os autores constataram que todos os estudos incorporaram o conceito de SEs para cumprir com os já citados requerimentos do IFC.

Estes resultados levantam a questão da importância da inserção dos SEs na etapa de escopo da AIA, a fim de identificar adequadamente os SEs que deverão ser abordados durante o processo. Caso a realização das tarefas nesta etapa seja falha, etapas subsequentes, como a análise dos impactos e a definição de medidas mitigadoras, podem ser prejudicadas (Landsberg et al., 2011; Rosa \& Sánchez, 2015).

De acordo com profissionais envolvidos na prática da AIA nos Estados Unidos, a exigência legal é citada como um dos fatores de encorajamento à 
incorporação do conceito de SEs na AIA (Presnall et al., 2015). Entretanto, há evidências de que, mesmo naqueles processos em que a análise dos SEs esteve inclusa no escopo, tarefas importantes foram omitidas ou mal executadas (Rosa \& Sánchez, 2015).

Alguns desafios científicos que precisam de solução têm sido apontados por Coleby et al. (2012) como entraves para a incorporação de uma abordagem ecossistêmica nos EIAs, tais como: a falta de conjuntos de dados sobre os serviços ecossistêmicos, o pouco entendimento de como os serviços respondem às intervenções humanas, a ausência de métricas para mensurar alguns benefícios ecossistêmicos considerados pelas populações, dentre outros. De fato, segundo profissionais de AIA nos Estados Unidos, um fator que estaria relacionado com a baixa incorporação dos SEs na avaliação é a falta de clareza e familiaridade do público e dos profissionais com relação ao conceito (Presnall et al., 2015). Além disso, também é citada a escassez de métodos e dados para a avaliação dos SEs (Honrado et al., 2013; Presnall et al., 2015). Apesar disto, uma abordagem no nível de SEs foi considerada por esses profissionais como potencialmente útil para melhorar a prática de AIA, em especial nas etapas de informação do público e dos tomadores de decisão (Presnall et al., 2015).

Nesse sentido, alguns estudos, guias e ferramentas já formulados podem ser utilizados ou adaptados para contribuir com a integração dos serviços ecossistêmicos na AIA. Além das orientações presentes nos Padrões de Desempenhos da IFC (IFC, 2012) e nas guias do WRI (Landsberg et al., 2013), trabalhos como os de Karjalainen et al. (2013) e Hooper et al. (2014) apresentam metodologias para apoiar a inserção dos SEs na avaliação de impacto, sendo que Hooper et al. (2014) focam nos impactos decorrentes de empreendimentos em escala local. Tallis et al. (2015) e Jacob et al. (2016) apresentam métodos focados na integração dos SEs na etapa de mitigação, sendo que Jacob et al. (2016) fornecem um método para auxiliar na escolha de alternativas compensatórias mais eficientes em maximizar a provisão de SEs. Em termos de ferramentas, Fürst et al. (2010) apresentam uma plataforma on-line que permite analisar os impactos decorrentes de alternativas de planejamento em múltiplos SEs em diversas escalas. Mandle et al. (2016) desenvolveram um software livre voltado para avaliar o impacto de um projeto nos SEs e determinar opções de mitigação mais eficientes. Tardieu et al. (2013; 2015) demonstram de forma prática como os SEs podem ser incorporados na avaliação de alternativas locacionais de projetos de infraestrutura de transporte terrestre. Os autores indicam que o mapeamento da perda de SEs de diferentes opções de traçado de uma rodovia foi agregado à análise custo-benefício dos projetos, inserindo os SEs na tomada de decisão (Tardieu et al., 2015).

Como resultado da segunda etapa, foram atribuídas pontuações para cada critério utilizado na análise de cada um dos EIAs que apresentaram o conceito de SEs, conforme a Tabela 2. Como se pode verificar, dentre as questões dos critérios de análise, cinco delas apresentaram nota zero para todos os EIAs. Desta forma, nenhum EIA dedicou um capítulo para analisar os ecossistemas afetados e seus serviços, e também nenhum delimita uma área de estudo para os SEs. Uma baseline para os SEs não é apresentada, sendo que não são previstos e analisados os impactos específicos nos SEs considerados prioritários, não havendo também medidas mitigadoras resultantes de uma análise sistemática da avaliação de impacto nos SEs. Estes resultados indicam que, ainda que citados implícita ou explicitamente, a incorporação dos SEs no processo de avaliação de impacto ainda é feita de forma incompleta (Jacob et al., 2016). 
TABELA 2 - Avaliações atribuídas a cada um dos critérios analisados, para cada um dos EIAs que incluíram pelo menos uma das quatro palavras-chave referentes a serviços ecossistêmicos entre 2006 e 2014

\begin{tabular}{|c|c|c|c|c|c|c|c|c|c|c|c|c|c|c|c|c|c|c|c|c|c|}
\hline & \multicolumn{20}{|c|}{ EIA } \\
\hline & & \multicolumn{4}{|c|}{2014} & \multicolumn{7}{|c|}{2013} & \multicolumn{4}{|c|}{2012} & \multirow{2}{*}{$\frac{\bar{\Xi}}{\stackrel{\sim}{\Xi}}$} & \multirow{2}{*}{$\frac{\text { હे }}{\text { ১ }}$} & \multicolumn{2}{|c|}{2008} & \multirow{2}{*}{ 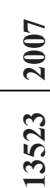 } \\
\hline & & $n$ & $\stackrel{M}{=}$ & $\cong$ & $\stackrel{\circ}{\text { f }}$ & $\because$ & $\infty$ & ळి & 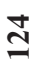 & $\stackrel{\infty}{\infty}$ & 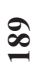 & 党 & ळे & ฉ & $\stackrel{\Xi}{\Xi}$ & $\infty$ & & & 3 & $\stackrel{\infty}{=}$ & \\
\hline \multirow{12}{*}{ 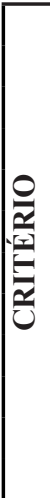 } & 1 & 0 & 0 & 0 & 0 & 0 & 0 & 0 & 0 & 0 & 0 & 0 & 0 & 0 & 0 & 0 & 0 & 0 & 0 & 0 & 0 \\
\hline & 2 & 0 & 0 & 0 & 0 & 0 & 0 & 1 & 0 & $\mathbf{0 , 5}$ & 1 & 0 & 0 & 0 & 0 & 0 & 0 & 0 & 0 & 0 & 0 \\
\hline & 3 & 0 & 0 & 0 & 0 & 0 & 0 & 0 & 0 & 0 & 0 & 0 & 0 & 0 & 0 & 0,5 & 0 & 0 & 0 & 0 & 0 \\
\hline & 4 & 0 & 0 & 0 & 0 & 0 & 0 & 0 & 0 & $\mathbf{0 , 5}$ & 0 & 0 & 0 & 0 & 0 & 0 & 0 & 0 & 0 & 0 & 0 \\
\hline & 5 & 0 & 0 & 0 & 0 & 0 & 0 & 0 & 0 & 0 & 0 & 0 & 0 & 0 & 0 & 0 & 0 & 0 & 0 & 0 & 0 \\
\hline & 6 & 0 & 0 & 0 & 0 & 0 & 0 & 0 & 0 & 0 & 0 & 0 & 0 & 0 & 0 & 0 & 0 & 0 & 0 & 0 & 0 \\
\hline & 7 & 0 & 0 & 0 & 0 & 0 & 0 & 0,5 & 0 & 0 & 0 & 0 & 0 & 0 & 0 & 0 & 0 & 0 & 0 & 0 & 0 \\
\hline & 8 & 0 & 0 & 0 & 0 & 0 & 0 & 0 & 0 & 0 & 0 & 0 & 0 & 0 & 0 & 0 & 0 & 0 & 0 & 0 & 0 \\
\hline & 9 & 0 & 0 & 0 & 0 & 0 & 0 & 1 & 0 & 0 & 0 & 0 & 0 & 0,5 & 0 & 0 & 0 & 0 & 0 & 0 & 0 \\
\hline & 10 & 0,5 & 0 & 0 & 0 & 0,5 & 0 & 1 & 1 & 1 & 1 & 1 & 0 & 0 & 0 & 1 & 0 & 0 & 0 & 0 & 0 \\
\hline & 11 & 0 & 0 & 0 & 0 & 0 & 0 & 0 & 0 & 0 & 0 & 0 & 0 & 0 & 0 & 1 & 0 & 0 & 0 & 0 & 0 \\
\hline & 12 & 0 & 0 & 0 & 0 & 0 & 0 & 0 & 0 & 0 & 0 & 0 & 0 & 0 & 0 & 0 & 0 & 0 & 0 & 0 & 0 \\
\hline
\end{tabular}

Legenda: Avaliações: 1 = Adequado; $0,5=$ Parcialmente adequado; 0 = Não citado ou não realizado.

Critérios: 1 . Os ecossistemas afetados estão descritos em um capítulo dedicado aos SEs?; 2. Há identificação dos SEs potencialmente afetados?; 3. Os beneficiários de cada SE estão descritos?; 4. Os SEs estão priorizados?; 5. Há uma delimitação da área de estudo para os SEs?; 6. Há uma baseline dedicada aos SEs prioritários?; 7. Há alguma forma de previsão dos impactos nos SEs?; 8. Os impactos nos SEs prioritários estão analisados?; 9. Existem medidas de mitigação indicadas para os SEs?; 10. Há menção dos SE em capítulos separados daquele dedicado aos SEs?; 11. Os diferentes tipos de SEs são explicitados em Serviços de Provisão, Serviços regulatórios, Serviços Culturais e Serviços de Suporte?; 12. Existem recomendações de mitigação resultando apenas da análise dos SEs?

De modo semelhante, Rosa \& Sánchez (2015) verificaram que nenhum dos cinco EIAs analisados descreveu os beneficiários dos SEs ou previu impactos nos mesmos. No presente trabalho, também se pôde constatar que essas demandas (critérios 3 e 7) foram fracamente atendidas. $\mathrm{Na}$ amostra analisada por Honrado et al. (2013), a maioria dos relatórios de impacto ambiental não quantificou ou mapeou os SEs. No presente trabalho, quadro semelhante também foi encontrado, pois apenas três EIAs cumpriram com a identificação dos SEs potencialmente afetados (critério 2). Portanto, ainda são desafios: a caracterização dos beneficiários dos SEs e a quan- tificação do suprimento dos SEs a fim de subsidiar a previsão dos impactos (Rosa \& Sánchez, 2015).

Foram então calculados os IASEs dos 20 EIAs que citaram pelo menos uma das palavras-chave. Os valores obtidos para cada um dos EIAs analisados, comparando-os e evidenciando o baixo valor apresentado por todos os estudos, estão apresentados na Figura 2.

Como se pode derivar dos dados fornecidos pela Figura 2, 11 EIAs apresentaram valor zero, indicando que nenhum dos 12 critérios de análise foi atendido. Os EIAs restantes apresentaram valores entre 0,04 e 0,29. Portanto, metade dos EIAs 


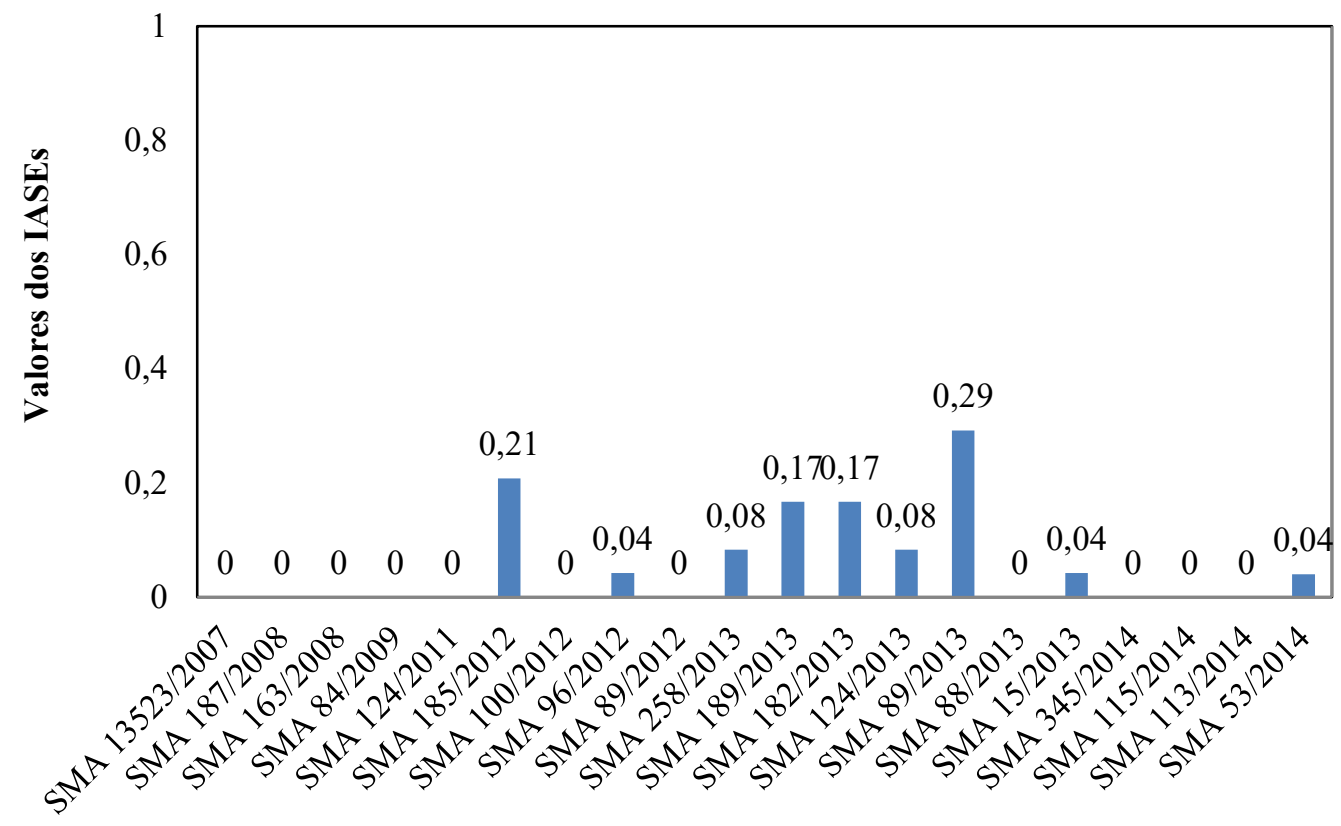

\section{Processos dos EIAs que incluíram palavras-chave referentes aos SEs}

FIGURA 2 - Comparação entre os valores dos IASEs obtidos para os EIAs que incluíram pelo menos uma das quatro palavras-chave referentes a serviços ecossistêmicos entre 2006 e 2014.

analisados (55\%) apresentaram IASE $=0$, ou seja, apesar de citar os termos relativos aos SEs, não há incorporação de fato destes conceitos na análise ambiental. Ademais, o EIA melhor pontuado (EIA 89 de 2013) apresentou IASE $=0,29$, indicando que, apesar de incluir algumas questões, a incorporação do conceito de SEs ainda é deficiente. Isso é refletido pelo número de critérios que foram parcial ou adequadamente atendidos por este EIA, somente quatro. Assim, este EIA identifica adequadamente os SEs potencialmente afetados pelo empreendimento, prevê possíveis impactos nos SEs identificados, ainda que de forma incompleta, e indica adequadamente medidas mitigadoras para esses impactos. Entretanto, as outras tarefas não foram trabalhadas.
Em geral, quando citados pelos EIAs, os SEs apareciam comumente vinculados às Unidades de Conservação (UCs), biomas ou à vegetação local direta ou indiretamente afetados. Isso indica que estas áreas têm sido reconhecidas como reduto de SEs, ou seja, estão sendo consideradas como essenciais na provisão dos serviços (Daily, 1997). Entretanto, esse reconhecimento se dá de forma limitada, pois são fornecidas apenas informações genéricas, não havendo um esforço sistemático para identificar os reais SEs que estas áreas proveem e que podem ser afetados pelo empreendimento.

Além disso, foi comum a inserção das palavras-chave no tópico de compatibilidade com políticas, planos, programas e projetos colocalizados, 
especificamente por meio da definição do Zoneamento Ecológico-Econômico (ZEE). Entretanto, essa informação geralmente aparece desvinculada da análise ambiental do EIA.

Alguns EIAs possuíam as palavras-chave inseridas no diagnóstico do meio, entretanto, foi comum que a informação provida não tenha sido utilizada explicitamente para identificar ou prever impactos. Rosa \& Sánchez (2015) também encontraram cenário semelhante para alguns casos analisados, onde havia uma baseline para os SEs considerados prioritários, mas esta não serviu de apoio para a previsão de impactos.

Finalmente, foi constatada uma confusão entre a definição do conceito que é utilizado neste trabalho e a definição que ocasionalmente apareceu nos EIAs. Alguns estudos tratavam "serviços ambientais" como sinônimos de serviços de saneamento, ou seja, por ocasião da instalação do empreendimento proposto haveria uma agregação de "serviços ambientais" à área.

\section{Conclusão}

Ao analisar os EIAs submetidos à CETESB, órgão ambiental responsável pelo licenciamento em São Paulo, pôde-se verificar que, de uma amostra de 110 estudos do período entre 2006 e 2014, 18\% (20 EIAs) empregaram explicitamente o conceito de SEs. Entre 2006 e 2010 foi verificada uma adoção instável do conceito, entretanto, a partir de 2011, nota-se claramente uma tendência de maior incorporação do mesmo pelos EIAs, sendo que, em
2014, 80\% dos EIAs submetidos apresentaram pelo menos uma das quatro palavras-chave. Como foi discutido, esta incorporação aparenta ser voluntária, visto que ainda não há exigências explícitas do órgão ambiental para que este tema seja trabalhado pelos estudos ambientais.

Apesar deste resultado, os SEs não foram incorporados de maneira adequada nos EIAs analisados. Os SEs foram fracamente identificados, poucos EIAs se dispuseram a identificar os possíveis impactos nestes serviços e nenhum estudo previu medidas mitigadoras. Desta forma, pode-se concluir que o conceito de serviços ecossistêmicos não se encontra incorporado à prática da AIA no estado de São Paulo, havendo necessidade de mais avanços na pesquisa sobre os SEs. Ademais, para que a incorporação dos SEs nos EIAs possa ser considerada adequada, se faz necessário o cumprimento de várias tarefas pelos estudos ambientais. Esses resultados corroboram a afirmação de que ainda é necessário um maior esforço político, normativo e técnico para o tratamento dos SEs na AIA (Honrado et al., 2013), pois há poucas evidências de contribuição da avaliação dos SEs à análise ambiental executada pelo EIA (Rosa \& Sánchez, 2015). Além disso, a implementação de uma abordagem em nível de SEs na AIA ainda enfrenta desafios que necessitam de pesquisas para serem solucionados (Coleby et al., 2012). Entretanto, há alguns recursos disponíveis que poderiam orientar a introdução dos SEs no processo de AIA, como guias de órgãos internacionais financiadores, metodologias conceituais e ferramentas de modelagem dos serviços ecossistêmicos. 


\section{Referências}

Atkinson, S. F.; Bhatia, S.; Schoolmaster, F. A.; Waller, W. T. Treatment of biodiversity impacts in a sample of US environmental impact statements. Impact Assessment and Project Appraisal, 18(4), 271-282, 2000. doi: 10.3152/147154600781767349

Baker, J.; Sheate, W. R.; Phillips, P.; Eales, R. Ecosystem services in environmental assessment - help or hindrance? Environmental Impact Assessment Review, 40, 3-13, 2013. doi: 10.1016/j.eiar.2012.11.004

CONAMA - Conselho Nacional do Meio ambiente. Resolução $n^{\circ}$ 01, de 23 de janeiro de 1986. Brasília: DOU de 17/02/1986.

CDB - Convenção sobre a Diversidade Biológica. Ecosystem approach. Disponível em: $<$ https://www.cbd.int/ecosystem/default.shtml>. Acesso em: set. 2015.

CETESB - Companhia Ambiental do Estado de São Paulo. Licenciamento ambiental: EIA/RIMA. Disponível em: $<\mathrm{http}$ ://icenciamentoambiental.cetesb.sp.gov.br/eia-rima/>. Acesso em: out. 2015.

CETESB - Companhia Ambiental do Estado de São Paulo. Manual para elaboração de estudos para o licenciamento com Avaliação de Impacto Ambiental, 2014. Disponível em: $<$ http://licenciamento.cetesb.sp.gov.br/cetesb/documentos/ Manual-DD-217-14.pdf>. Acesso em: out. 2015.

Coleby, A.; Van Der Horst, D.; Hubacek, K.; Goodier, C.; Burgess, P. J.; Graves, A.; Lord, R.; Howard, D. Environmental Impact Assessment, ecosystems services and the case of energy crops in England. Journal of Environmental Planning and Management, 55(3), 369-385, 2012. doi: 10.1080/09640568.2011.603958

Conesa Fernández-Vítora, V. Guía metodológica para la evaluación del impacto ambiental. Madrid: Mundi-Prensa, 2006. Disponível em: <http://centro.paot.mx/documentos/ varios/guia_metodologica_impacto_ambiental.pdf $>$. Acesso em: jul. 2017.

Daily, G. C. Nature's services: societal dependence on natural ecosystems. Washington, DC: Island, 1997. 392 p.

Daily, G. C.; Polasky, S.; Goldstein, J.; Kareiva, P. M.; Mooney, H. A.; Pejchar, L.; Ricketts, T. H.; Salzman, J.; Shallenberger, R. Ecosystem services in decision making: time to deliver. Frontiers in Ecology and the Environment, 7(1), 21-28, 2009. doi: 10.1890/080025

Fürst, C.; Volk, M.; Pietzsch, K.; Makeschin, F. Pimp Your Landscape: A Tool for Qualitative Evaluation of the Effects of Regional Planning Measures on Ecosystem Services. Environmental Management, 46(6), 953-968, 2010. doi: 10.1007/s00267-010-9570-7

Geneletti, D. Ecosystem services in environmental impact assessment and strategic environmental assessment. Environmental Impact Assessment Review, 40, 1-2, 2013. doi: 10.1016/j.eiar.2013.02.005

Glasson, J.; Therivel, R.; Chadwick, A. Introduction to Environmental Impact Assessment. 4th ed. Abingdon: Routledge, 2012.

Honrado, J. P.; Vieira, C.; Soares, C.; Monteiro, M. B.; Marcos, B.; Pereira, H. M.; Partidário, M. R. Can we infer about ecosystem services from EIA and SEA practice? A framework for analysis and examples from Portugal. Environmental Impact Assessment Review, 40, 14-24, 2013. doi: 10.1016/j.eiar.2012.12.002

Hooper, T.; Cooper, P.; Hunt, A.; Austen, M. A methodology for the assessment of local-scale changes in marine environmental benefits and its application. Ecosystem Services, 8, 65-74, 2014. doi:10.1016/j.ecoser.2014.02.005

IAIA - International Association for Impact Assessment. What Is Impact Assessment?, 2009. Disponível em: $<$ http:// www.iaia.org/uploads/pdf/What_is_IA_web.pdf $>$. Acesso em: jul. 2017.

IFC - International Finance Corporation. Performance standards on environmental and social sustainability. Washington, DC: IFC, 2012.

Jacob, C.; Vaissiere, A.; Bas, A.; Calvet, C. Investigating the inclusion of ecosystem services in biodiversity offsetting. Ecosystem Services, 21, 92-102, 2016. doi: 10.1016/j. ecoser.2016.07.010

Karjalainen, T. P.; Marttunen, M.; Sarkki, S.; Rytkönen, A. Integrating ecosystem services into environmental impact assessment: An analytic-deliberative approach. Environmental Impact Assessment Review, 40, 54-64, 2013. doi: 10.1016/j.eiar.2012.12.001 
Landsberg, F.; Ozment, S.; Mercedes-Stickler, M.; Henninger, N.; Treweek, J.; Venn, O.; Mock, G. Ecosystem Services Review for Impact Assessment: Introduction and Guide to Scoping. WRI Working Paper. Washington, DC: World Resources Institute, 2011.

Landsberg, F.; Treweek, J.; Mercedes-Stickler, M.; Henninger, N.; Venn, O. Weaving ecosystem services into impact assessment: A step-by-step method. Abbreviated version 1.0.2013. Washington, DC: World Resources Institute, 2013.

Laurans, Y.; Rankovic, A.; Billé, R.; Pirard, R.; Mermet, L. Use of ecosystem services economic valuation for decision-making: Questioning a literature blindspot. Journal of Environmental Management, 119, 208-219, 2013. doi: 10.1016/j.jenvman.2013.01.008

Mandle, L.; Douglass, J.; Lozano, J.S.; Sharp, R.P.; Vogl, A.L.; Denu, D.; Walschburger, T.; Tallis, H. OPAL: An open-source software tool for integrating biodiversity and ecosystem services into impact assessment and mitigation decisions. Environmental Modelling \& Software, 84, 121133, 2016. doi: 10.1016/j.envsoft.2016.06.008

MEA - Millennium Ecosystem Assessment. Ecosystems and Human Well-being: Synthesis. Washington, DC: Island Press, 2005a.

MEA - Millennium Ecosystem Assessment. Ecosystems and Human Well-being: Health Synthesis. Washington, DC: Island Press, 2005b.

Presnall, C.; López-Hoffman, L.; Miller, M. L. Adding ecosystem services to environmental impact analyses: More sequins on a "bloated Elvis" or rockin' idea? Eco- logical Economics, 115, 29-38, 2015. doi: 10.1016/j. ecolecon.2014.02.001

Rosa, J. C. S.; Sánchez, L. E. Is the ecosystem service concept improving impact assessment? Evidence from recent international practice. Environmental Impact Assessment Review, 50, 134-142, 2015. doi: 10.1016/j.eiar.2014.09.006

Rosa, J. C. S.; Sánchez, L. E. Advances and challenges of incorporating ecosystem services into impact assessment. Journal of Environmental Management, 180, 485-492, 2016. doi: 10.1016/j.jenvman.2016.05.079

Sánchez, L. E. Avaliação de impacto ambiental: conceitos e métodos. 2. ed. São Paulo: Oficina de Textos, 2013.

Tallis, H.; Kennedy, C. M.; Ruckelshaus, M.; Goldstein, J.; Kiesecker, J. M. Mitigation for one \& all: An integrated framework for mitigation of development impacts on biodiversity and ecosystem services. Environmental Impact Assessment Review, 55, 21-34, 2015. doi: 10.1016/j. eiar.2015.06.005

Tardieu, L.; Roussel, S.; Salles, J. Assessing and mapping global climate regulation service loss induced by Terrestrial Transport Infrastructure construction. Ecosystem Services, 4, 73-81, 2013. doi: 10.1016/j.ecoser.2013.02.007

Tardieu, L.; Roussel, S.; Thompson, J. D.; Labarraque, D.; Salles, J. Combining direct and indirect impacts to assess ecosystem service loss due to infrastructure construction. Journal of Environmental Management, 152, 145-157, 2015. doi: 10.1016/j.jenvman.2015.01.034

Wunder, S. Payments for environmental services: some nuts and bolts. CIFOR Occasional Paper, 42, 1-24, 2005. 